\title{
A MATHEMATIC MODEL OF TWO MUTUALLY INTERACTING SPECIES WITH MORTALITY RATE FOR THE SECOND SPECIES
}

\author{
Annisa Rahayu, Yuni Yulida, Faisal \\ Department of Mathematics, Faculty of Mathematics and Natural Sciences \\ Lambung Mangkurat University, Banjarbaru, Indonesia \\ Email: chachanain@yahoo.co.id \\ y yulida@unlam.ac.id \\ isal faisal@unlam.ac.id
}

\begin{abstract}
One of the interactions that occur within the ecosystem is the interaction of mutualism. Such mutualism interactions can be modeled into mathematical models. Reddy (2011) study suggests a model of two mutually interacting species that assumes that each species can live without its mutualism partner. In fact, not all mutual species survive without their mutualism pairs. If it is assumed that the second species lives without its mutualism partner, the first species, then the natural growth rate of the second species population will decrease (the mortality rate). The purpose of this research is to explain the model of two mutually interacting species with mortality rate for the second species, to determine the equilibrium point and the type of stability, and to simulate them with several parameters. This research was done by way of literature studies. The result of this research is the model of two mutually interacting species with mortality rate for second species modeled using Nonlinear Differential Equation System. In the model, it was obtained 3 (three) points of equilibrium, with each type and type of stability investigated. Next up from the stability, model simulations were done. Based on several simulations conducted can be seen the value of parameters and initial values affect the population growth of both species. The interaction model of two mutual species will be stable if the first species survive and the second species over time will be extinct.
\end{abstract}

Keywords: Equilibrium Point, Interaction Model of Two Mutual Species, Type of Stability

\section{INTRODUCTION}

Ecology is one branch of biological science that studies about several populations in an ecosystem. One of the interactions that occur within the ecosystem is the interaction of mutualism. Such mutualism interactions can be modeled into mathematical models.
In the study of Reddy (2011), the mathematical model is the interaction model of two mutual species that assumes that the populations of the first species with limited resources and for the populations of the second species with unlimited resources. Each species benefits from the interaction that occur, but the survival of each species does not depend on mutual interaction. In this case, each species can live without its mutualism partner. In fact, not all mutual species survive without their mutualism pairs. If it is assumed that the survival of the first species does not depend on the interaction, while the survival of the second species depends on the interaction of mutualism, in this case the mutualism pair of the second species is the first species. Consequently, if the second species live without a mutualism partner, then the natural growth rate of the second species population will decrease (the mortality rate). Reddy, et. al. (2012) later on modified the model.

This article will explain how the interaction model of two mutual species with mortality rate for the second species, how to determine the equilibrium point and the type of stability model of the interaction of two mutual species with mortality rate for the second species, and how to make a model simulation with several parameters.

\section{METHOD}

This research was conducted by literature studies from various sources, both books and journals that support and relevant to the research conducted. The steps are to explain the formation of the model, to determine the equilibrium point, to investigate the local stability using model linearization (Jacobian Matrix), from the Jacobian Matrix, characteristic equation and eigenvalues were obtained. Based on the obtained eigenvalues, the type and type of stability can be determined. 


\section{LITERATURE REVIEW}

\section{System of Linear and Nonlinear Differential Equations of First Order}

The form of a homogeneous first-order linear differential equation system can be written as follows:

$$
\frac{d x}{d t}=A x
$$

with $x=\left(x_{1}, x_{2}, \ldots, x_{n}\right) \in R^{n}$

$$
\begin{gathered}
\frac{d \boldsymbol{x}}{d t}=\left[\begin{array}{c}
\frac{d x_{1}}{d t} \\
\frac{d x_{2}}{d t} \\
\vdots \\
\frac{d x_{n}}{d t}
\end{array}\right] \\
\text { and } A=\left[\begin{array}{cccc}
a_{11} & a_{12} & \cdots & a_{1 n} \\
a_{21} & a_{22} & \cdots & a_{2 n} \\
\vdots & \vdots & & \vdots \\
a_{n 1} & a_{n 2} & \cdots & a_{n n}
\end{array}\right]
\end{gathered}
$$

Given a system of first-order nonlinear differential equations as follows:

$$
\frac{d \boldsymbol{x}}{d t}=\boldsymbol{f}(t, \boldsymbol{x})
$$

with $\boldsymbol{x}=\left(x_{1}, x_{2}, \ldots, x_{n}\right) \in R^{n}$ and $\boldsymbol{f}(t, \boldsymbol{x}) \in$ $R^{n} . \boldsymbol{f}(t, \boldsymbol{x})$ as a nonlinear function. [3]

\section{Equilibrium Point}

The definition of an equilibrium point can be written as follows.

\section{Definition 3.3.1 [4]}

Point $\hat{\boldsymbol{x}} \in R^{n}$ is called the equilibrium point of the equation (3) if $\boldsymbol{f}(t, \hat{\boldsymbol{x}})=\mathbf{0}$.

\section{Linearization of Nonlinear Equation Systems}

To determine the stability of a nonlinear differential equation system at the equilibrium point $(\hat{\boldsymbol{x}})$, the nonlinear differential equations system needs to be linearized using a Jacobian matrix. The Jacobian matrix can be written as follows

$$
J(\hat{\boldsymbol{x}})=\left[\begin{array}{ll}
\frac{\partial f_{1}\left(t, \hat{x}_{1}, \hat{x}_{2}\right)}{\partial x_{1}} & \frac{\partial f_{1}\left(t, \hat{x}_{1}, \hat{x}_{2}\right)}{\partial x_{2}} \\
\frac{\partial f_{2}\left(t, \hat{x}_{1}, \hat{x}_{2}\right)}{\partial x_{1}} & \frac{\partial f_{2}\left(t, \hat{x}_{1}, \hat{x}_{2}\right)}{\partial x_{2}}
\end{array}\right]
$$

The Jacobian matrix will then be used to determine the eigenvalues of the linear equation system. [2]

\section{Eigenvalues and Eigenvectors}

The following are the definitions of eigenvalues and eigenvectors.

\section{Definition 3.5.1 [1]}

If $\mathrm{A}$ is $n \times n$ matrix, then the nonzero vector $\mathrm{x}$ in $R^{n}$ is called the eigenvector of $A$. If $A x$ is scalar multiplication from $\mathbf{x}$; namely, $A \mathbf{x}=\lambda \mathbf{x}$. Scalar $\lambda$ called the eigenvalue of $A$ and $\mathbf{x}$ are said to be a corresponding eigenvector to $\lambda$.

\subsection{System Stability}

\section{Theorem 3.6.1 [9]}

Let $\lambda_{j}(j=1,2, \ldots, n)$ to be the eigenvalue of the matrix $A$ in equation (2) and $\operatorname{Re}\left(\lambda_{\mathrm{j}}\right)$ is the real part from $\lambda_{j}$, then the equilibrium point $\hat{\boldsymbol{x}}$ is said to be:

(i) Stable if $\operatorname{Re}\left(\lambda_{\mathrm{j}}\right) \leq 0$ and at least one eigenvalue of the matrix $A$ real part is zero.

(ii) Asymptotically stable if $\operatorname{Re}\left(\lambda_{\mathrm{j}}\right)<0$.

(iii) Unstable if there is an eigenvalue of the matrix $A$ with positive real part.

Table 1. Linear System Stability [5]

\begin{tabular}{ccc}
\hline Eigenvalues & Type of Equilibrium Point & Stability \\
\hline $\begin{array}{c}\lambda_{1}>\lambda_{2}>0 \\
\lambda_{1}=\lambda_{2}>0\end{array}$ & Node & Unstable \\
$\lambda_{1}<\lambda_{2}<0$ & & \\
$\lambda_{1}=\lambda_{2}<0$ & Node & Asymptotically stable \\
$\lambda_{2}<0<\lambda_{1}$ & Saddle & Unstable \\
$\lambda_{1}, \lambda_{2}=a \pm i b$ & & \\
$a>0$ & Spiral & Unstable \\
$a<0$ & & Asymptotically stable \\
$\lambda_{1}, \lambda_{2}= \pm i b$ & Center & Stable but asymptotically unstable \\
$($ Purely Imaginary $)$ & &
\end{tabular}




\section{RESULT DAN DISCUSSION}

Models of Two Mutually Interacting Species With Mortality Rate for The Second Species

Let $\mathrm{N}_{1}(t)$ denote the number of the first species in the population at the time $t$ and $\mathrm{N}_{2}(t)$ denote the number of the second species in the population at the time $t$. The assumptions used to build the model as follows:

1. The natural growth in the first species population in the absence of the second species will increase exponentially.

2. There is interaction among the first species competing for limited resources.

3. The mutualism pair of the second species is the first species.

4. The natural growth of the second species population in the absence of a mutualism partner will decrease exponentially.

5. The source of food and living space of the second species depends on its mutualism partner, the first species, resulting in the interaction between the first species and the second species.

6. Interaction that occurs between the first species and the second species provide benefits for each species.

Based on the above assumptions, a model of two mutually interacting species with mortality rate for the second species is as follows.

$$
\begin{aligned}
& \qquad \begin{array}{l}
\frac{d N_{1}}{d t}=a_{1} N_{1}-\alpha_{11} N_{1}^{2}+\alpha_{12} N_{1} N_{2} \\
\frac{d N_{2}}{d t}=-a_{2} N_{2}+\alpha_{21} N_{1} N_{2} \\
\text { With } 0 \leq a_{1} \leq 1,0 \leq a_{2} \leq 1 \\
0 \leq \alpha_{11} \leq 1,0 \leq \alpha_{12} \leq 1, \text { and } 0 \leq \alpha_{21} \leq 1
\end{array}
\end{aligned}
$$

Some parameters used in the model are as follows.

$a_{1}=$ the natural growth rate of the first species population.

$a_{2}=$ the natural mortality rate of the second species.

$\alpha_{11}=$ the rate of the first species population decline due to interaction in the first species in the first species population.

$\alpha_{12}=$ the growth rate of the first species population because it interacts with the second species.

$\alpha_{21}=$ the rate of population growth of the second species by interacting with the first species.

\section{The Model Equilibrium Point}

By the definition 3.3.1, Model [7] must meet $\frac{d N_{1}}{d t}=0$ and $\frac{d N_{2}}{d t}=0$ which result three equilibrium points on the model of two mutually interacting species are obtained namely

$$
\begin{aligned}
& \text { I. } \quad\left(\hat{N}_{1}, \hat{N}_{2}\right)=(0,0) \\
& \text { II. } \quad\left(\hat{N}_{1}, \hat{N}_{2}\right)=\left(\frac{a_{1}}{\alpha_{11}}, 0\right) \\
& \text { III. }\left(\hat{N}_{1}, \hat{N}_{2}\right)=\left(\frac{a_{2}}{\alpha_{21}}, \frac{a_{2} \alpha_{11}-a_{1} \alpha_{21}}{\alpha_{12} \alpha_{21}}\right) \text { with the } \\
& \text { provision of } a_{2} \alpha_{11}-a_{1} \alpha_{21}>0 .
\end{aligned}
$$

\subsection{System Stability}

\section{System Stability at Equilibrium Point I}

Jacobian matrix at equilibrium point

$$
\begin{aligned}
& \left(\hat{N}_{1}, \hat{N}_{2}\right)=(0,0) \text { is } \\
& J(0,0)=\left[\begin{array}{rr}
a_{1} & 0 \\
0 & -a_{2}
\end{array}\right]
\end{aligned}
$$

The characteristic equation of (8) is as follows.

$$
\left(a_{1}-\lambda\right)\left(-a_{2}-\lambda\right)=0
$$

From equation (9) is obtained $\lambda_{1}=a_{1}>0$ and $\lambda_{2}=-a_{2}<0$.

Because and $\lambda_{2}<0$, then based on Theorem 3.6.1 The model is unstable at the point $(0,0)$ and is a saddle point type. Simulation of the equilibrium point can be seen in the figure 1. From this point it cannot be concluded, because the equilibrium point is unstable.

\section{System Stability at Equilibrium Point II} Jacobian matrix at equilibrium point

$$
\begin{aligned}
& \left(\hat{N}_{1}, \hat{N}_{2}\right)=\left(\frac{a_{1}}{\alpha_{11}}, 0\right) \text { is } \\
& J\left(\frac{a_{1}}{\alpha_{11}}, 0\right)=\left[\begin{array}{cc}
-a_{1} & \frac{a_{1} \alpha_{12}}{\alpha_{11}} \\
0 & -a_{2}+\frac{a_{1} \alpha_{21}}{\alpha_{11}}
\end{array}\right]
\end{aligned}
$$

The characteristic equation of (10) is as follows.

$$
\left(-a_{1}-\lambda\right)\left(-a_{2}+\frac{a_{1} \alpha_{21}}{\alpha_{11}}-\lambda\right)=0
$$

From equation (11) is obtained $\lambda_{1}$ and

$\lambda_{2}$ as follows.

$$
\begin{aligned}
& \lambda_{1}=-a_{1}<0, \text { or } \lambda_{2}=\frac{a_{1} \alpha_{21}}{\alpha_{11}}-a_{2}, \text { with } \\
& a_{1}, a_{2}, \alpha_{11}, \alpha_{21}>0 .
\end{aligned}
$$


Based $\lambda_{1}$ and $\lambda_{2}$ above, then we can know the stability of the system at the equilibrium point $\left(\frac{a_{1}}{\alpha_{11}}, 0\right)$ are as follows.

(i) If $\frac{a_{1}}{\alpha_{11}}>\frac{a_{2}}{\alpha_{21}}$, then $\lambda_{1}<0$ and $\lambda_{2}>0$, so that by Theorem 3.6.1 Model (7) is unstable at this equilibrium point and from Table 1 the type is of saddle point. The simulation of this equilibrium point can be seen in Figure 2.

(ii) If $\frac{a_{1}}{\alpha_{11}}<\frac{a_{2}}{\alpha_{21}}$ then $\lambda_{1}<0$ dan $\lambda_{2}<0$, so that based on Theorem 3.6.1 Model (7) is asymptotically stable at this point of equilibrium and from Table 7 the type is of node point. The simulation of this equilibrium point can be seen in Figure 3 . This situation explains that the first species population will survive in its habitat while the second species will be destroyed over time.

\section{System Stability at Equilibrium Point III} Jacobian matrix at equilibrium point $\left(\hat{N}_{1}, \hat{N}_{2}\right)=\left(\frac{a_{2}}{\alpha_{21}}, \frac{a_{2} \alpha_{11}-a_{1} \alpha_{21}}{\alpha_{12} \alpha_{21}}\right)$ is $J\left(\frac{a_{2}}{\alpha_{21}}, \frac{a_{2} \alpha_{11}-a_{1} \alpha_{21}}{\alpha_{12} \alpha_{21}}\right)=\left[\begin{array}{cc}-\frac{a_{2} \alpha_{11}}{\alpha_{21}} & \frac{a_{2} \alpha_{12}}{\alpha_{21}} \\ \frac{a_{2} \alpha_{11}-a_{1} \alpha_{21}}{\alpha_{12}} & 0\end{array}\right]$
The characteristic equation of (12) is as follows.

$$
\lambda^{2}+\frac{a_{2} \alpha_{11}}{\alpha_{21}} \lambda-\frac{a_{2}\left(a_{2} \alpha_{11}-a_{1} \alpha_{21}\right)}{\alpha_{21}}=0
$$
as follows.

The discriminant (D) of equation (13) is $D=\left(\frac{a_{2} \alpha_{11}}{\alpha_{21}}\right)^{2}+\frac{4 a_{2}\left(a_{2} \alpha_{11}-a_{1} \alpha_{21}\right)}{\alpha_{21}}>0$, provision $a_{2} \alpha_{11}-a_{1} \alpha_{21}>0$

Consequently $\mathrm{D}>0$, so equation (13) has two distinct real roots. The roots of equation (13) are as follows.

$\lambda_{1}=-\frac{1}{2}\left(\frac{a_{2} \alpha_{11}}{\alpha_{21}}\right)+\frac{1}{2} \sqrt{D}, \quad D>0$ and $\sqrt{D}>\frac{a_{2} \alpha_{11}}{\alpha_{21}}$, so that $\lambda_{1}>0$.

$\lambda_{2}=-\frac{1}{2}\left(\frac{a_{2} \alpha_{11}}{\alpha_{21}}\right)-\frac{1}{2} \sqrt{D}, D>0$ so that $\lambda_{2}<0$.

Based on Theorem 3.6.1 then Model (7) is unstable at this equilibrium point and the type of equilibrium point is the saddle point. The simulation of this equilibrium point can be seen in Figure 4. This shows that both species cannot be explained through this point.

\subsection{Simulation}

Table 2. Initial value and some parameter values

\begin{tabular}{lcccccccc}
\hline & Equilibrium Point & $N_{1}(t=0)$ & $N_{2}(t=0)$ & $a_{1}$ & $\alpha_{11}$ & $\alpha_{12}$ & $a_{2}$ & $\alpha_{21}$ \\
\hline I & & 70 & 10 & 0.5 & 0.004 & 0.002 & 0.3 & 0.003 \\
II & Case 1 & 50 & 1 & 0.3 & 0.01 & 0.001 & 0.2 & 0.01 \\
III & Case 2 & 50 & 1 & 0.3 & 0.03 & 0.01 & 0.1 & 0.006 \\
\hline
\end{tabular}

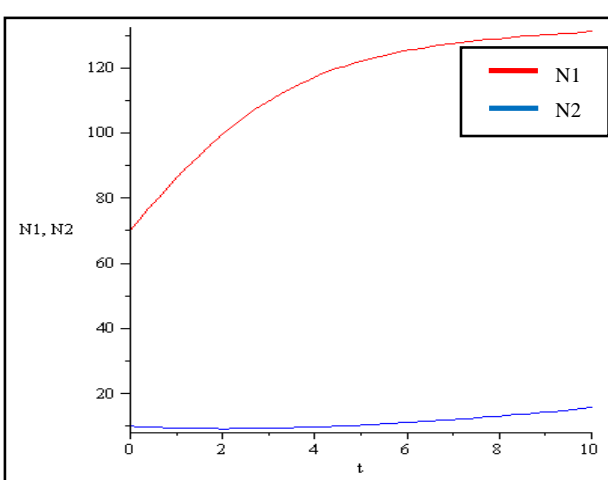

Figure 1. Simulation of Equilibrium Point I Case 1

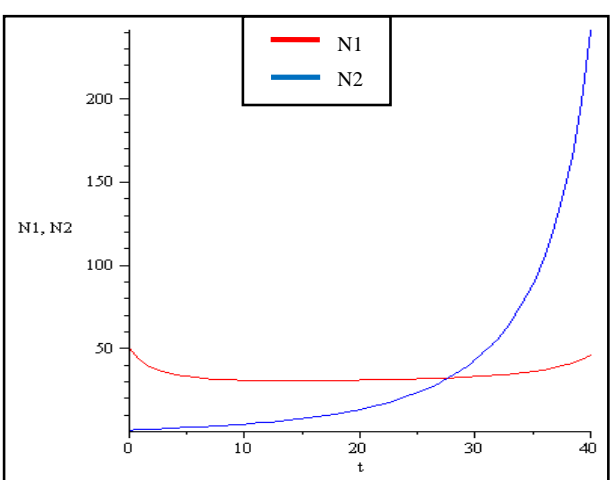

Figure 2. Simulation of Equilibrium Point II on 


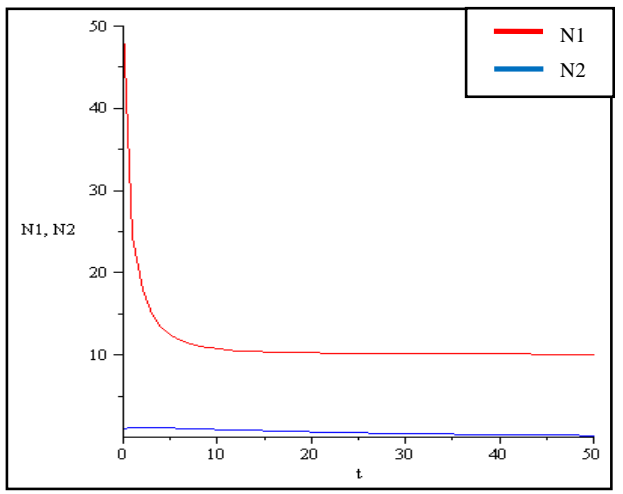

Figure 3. Simulation of Equilibrium Point II on Case 2

\section{CONCLUSIONS}

The conclusion obtained from this research is the interaction model of two mutual species can be written $\frac{d N_{1}}{d t}=a_{1} N_{1}-\alpha_{11} N_{1}^{2}+$ $\alpha_{12} N_{1} N_{2}$ and $\frac{d N_{2}}{d t}=-a_{2} N_{2}+\alpha_{21} N_{1} N_{2}$.

In the interaction model of two mutual species three equilibrium points are obtained $(0,0),\left(\frac{a_{1}}{\alpha_{11}}, 0\right)\left(\frac{a_{2}}{\alpha_{21}}, \frac{a_{2} \alpha_{11}-a_{1} \alpha_{21}}{\alpha_{12} \alpha_{21}}\right)$.

The stability of the system at the equilibrium point in the interaction model of two mutual species is the unstable system at the equilibrium point $(0,0)$ and the type of equilibrium point is the saddle point. If $\frac{a_{1}}{\alpha_{11}}>\frac{a_{2}}{\alpha_{21}}$, then the system is unstable at the equilibrium point $\left(\frac{a_{1}}{\alpha_{11}}, 0\right)$, and the type of point of the equilibrium is the saddle point.

If $\frac{a_{1}}{\alpha_{11}}>\frac{a_{2}}{\alpha_{21}}$ then the system is asymptotically stable at the equilibrium point $\left(\frac{a_{1}}{\alpha_{11}}, 0\right)$, and the type of equilibrium point is the node point.

The system is unstable at the equilibrium point $\left(\frac{a_{2}}{\alpha_{21}}, \frac{a_{2} \alpha_{11}-a_{1} \alpha_{21}}{\alpha_{12} \alpha_{21}}\right)$ and the type of point of the equilibrium is the saddle point.

Based on several simulations conducted can be seen the value of parameters and initial values affect the population growth of both species. The interaction model of two mutual species will be stable if the first species survive and the second species over time will be extinct.

\section{REFERENCES}

Anton, H. (1994). Linear Algebra Elementer, Fifth Edition. Erlangga. Jakarta.

Bellomo, N. \& Preziosi, L. (1995). Modelling Mathematical Methods and Scientific Computation. CRC Press. Florida.

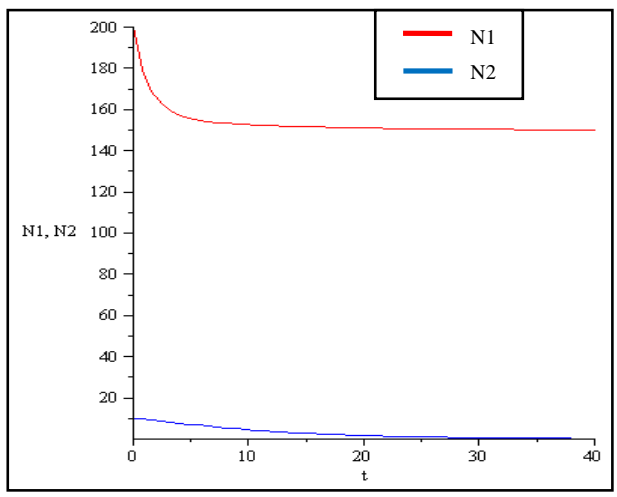

Figure 4. Simulation of Equilibrium Point III

Braun, M. (1992). Differential Equation and Their Applications-Fourth Edition. Springer-Verlag. New York.

Engwerda, J. (2005). LQ Dynamic Optimization and Differential Games. John Wiley \& Sons Inc. England.

Farlow, S. J. (1994). Differential Equations and Their Applications. Dover Publications. United States of America.

Reddy, B. R. (2012). A model of two mutually interacting species with mortality rate for the second species. Pelagia Research Library, Advances in Applied Science Research 3 (2): 757764.

Reddy, B.R., Kumar, N.P. \& Pattabhriramacharyulu, N.Ch. (2011). A model of mutually interacting species with limited resources of first species and unlimited for second species. ARPN Journal of Engineering and Applied Sciences 6 (1): 61-66.

Ross, S. L. (1984). Differential Equation-Third Edition. John Wiley \& Sons, Inc. New York.

Sanchez, D. A. (1968). Differential Equation and Stability Theory: An Introduction. W. H. Freeman and Company. 